\title{
Photophysical, Thermal and Structural Properties of Thiophene and Benzodithiophene-Based Copolymers Synthesized by Direct Arylation Polycondensation Method
}

\author{
Newayemedhin A. Tegegne ${ }^{1, * \mathbb{D}}$, Zelalem Abdissa ${ }^{2}$ and Wendimagegn Mammo ${ }^{2}$ \\ 1 Department of Physics, Addis Ababa University, Addis Ababa P.O. Box 1176, Ethiopia \\ 2 Department of Chemistry, Addis Ababa University, Addis Ababa P.O. Box 1176, Ethiopia; \\ zelalem2ab@yahoo.com (Z.A.); wendimagegn.mammo@aau.edu.et (W.M.) \\ * Correspondence: newaye.medhin@aau.edu.et
}

check for updates

Citation: Tegegne, N.A.; Abdissa, Z.; Mammo, W. Photophysical, Thermal and Structural Properties of

Thiophene and BenzodithiopheneBased Copolymers Synthesized by Direct Arylation Polycondensation Method. Polymers 2021, 13, 1151. https://doi.org/10.3390/ polym13071151

Academic Editor: Edina Rusen

Received: 28 February 2021

Accepted: 22 March 2021

Published: 4 April 2021

Publisher's Note: MDPI stays neutra with regard to jurisdictional claims in published maps and institutional affiliations.

Copyright: (c) 2021 by the authors. Licensee MDPI, Basel, Switzerland. This article is an open access article distributed under the terms and conditions of the Creative Commons Attribution (CC BY) license (https:// creativecommons.org/licenses/by/ $4.0 /)$
Abstract: Three low-band-gap copolymers based on isoindigo acceptor units were designed and successfully synthesized by direct arylation polycondensation method. Two of them were benzodithiophene (BDT)-isoindigo copolymers (PBDTI-OD and PBDTI-DT) with 2-octlydodecyl (OD) and 2-decyltetradecyl (DT) substituted isoindigo units, respectively. Thiophene donor and DTsubstituted isoindigo acceptor units were copolymerized to synthesize PTI-DT. The copolymers have a broad absorption range that extends to over $760 \mathrm{~nm}$ with a band gap $\approx 1.5 \mathrm{eV}$. The photophysical property studies showed that the BDT-based copolymers have non-polar ground states. Their emission exhibited the population of the intramolecular charge transfer (ICT) state in polar solvents and tightly bound excitonic state in non-polar solvents due to self-aggregation. On the contrary, the emission from the thiophene-based copolymers was only from the tightly bound excitonic state. The thermal decomposition temperature of the copolymers was above $380^{\circ} \mathrm{C}$. The $\mathrm{X}$-ray diffraction pattern of the three copolymers showed a halo due to $\pi-\pi$ stacking. A second, sharper peak was observed in the BDT-based copolymer with a longer side chain on the isoindigo unit (PBDTI-DT), and the thiophene-based copolymers with PTI-DT, exhibiting a better structural order.

Keywords: intramolecular charge transfer; copolymers; $\pi-\pi$ stacking; direct arylation polycondensation; and excitonic state

\section{Introduction}

The introduction of $\pi$-conjuageted polymers to the fast-growing solar cell technology has brought forth new features of flexibility and solution processabiltiy in the so-called organic solar cells (OSCs) devices. OSCs have shown a leap in efficiency in the last five years to above $18 \%$ in single-junction [1], and over 17\% in multi-junction [2], devices. This tremendous improvement is mainly attributed to the introduction of new donor polymers and non-fullerene acceptors that can harvest the broad solar spectrum with appropriate energetics in the active layer. A vast number of polymers have been synthesized and characterized to enrich the structure-property relation recipe for efficient OSCs. Alternating donor-acceptor (D-A) copolymers are commonly synthesized to obtain low-band-gap polymers with good solar harvest that extends to the near-infra-red region. Some important parameters that need to be addressed during the synthesis of the D-A copolymers include the HOMO level, which should be lower-lying, with the LUMO lying in the region for efficient exciton dissociation.

The synthesis of low-band-gap polymers using a simple and economically viable technique has gained the attention of researchers due to its potential for industrialization of the OSC technology. The direct arylation polycondensation method (DAP) has notably shortened the synthesis route compared to the commonly used Suzuki and Stille crosscoupling techniques. In this reaction, the unsubstituted arylene monomers are directly 
coupled with dihaloarylene units with the help of transition metals-usually palladium acetate. The by-product at each of the synthesis steps is, therefore, drastically reduced, making the DAP method more environmentally friendly and economically viable. In addition, the DAP method made the synthesis of polymers that were difficult to prepare with the traditional Suzuki and Stille cross-coupling techniques possible [3]. The mass production of OSCs will need an enormous amount of donor and acceptor organic materials. An easy and fast synthesis method such as DAP is undoubtedly important in the realization of the commercialization of OSC technology. Despite its many interesting merits, the method has a drawback: its selectivity between $\mathrm{C}-\mathrm{H}$ bonds for some monomers is poor. Consequently, the method is more commonly used to synthesize homopolymers like poly-3-hexylthiophene (P3HT) and benzodithiophene (BDT) [4-6]. However, D-A copolymers synthesized by DAP method are limited. Recently, our group and others have reported the successful synthesis of D-A copolymers using the DAP method [7-10].

The choice of $\mathrm{D}$ and $\mathrm{A}$ units in the synthesis of $\mathrm{D}-\mathrm{A}$ copolymers determines their electrical, optical and structural properties, which that will subsequently determine the performance of the OSC that the materials are used for. Isoindigo is a widely used acceptor unit due to its good electron-withdrawing properties. The fused ring benzo[1,2-b:4,5$\mathrm{b}^{\prime}$ ]dithiophene (BDTs) and thiophene units are the most commonly used donor units in the synthesis of D-A copolymers that produced high power conversion efficeny (PCE) of over $11 \%$ in OSCs [11]. These polymers are usually contain aloxy or alkyl side chains to enhance their solubility. A basic understanding of the structure-property relationships of the polymers is vital for the optimization of their structure for higher PCE of OSCs. The optical properties, intra/inter-chain interactions in the polymers, and their morphologies in the films are some of the parameters that play important roles in the photogeneration of charges in OSCs. In addition, their thermal properties determine their lifetimes in harsh environment conditions, and also their application in flexible OSC devices, which are normally processed at temperatures below $150^{\circ} \mathrm{C}$.

In this work, we report the synthesis of three D-A copolymers based on an isoindigo acceptor unit. The two copolymers have alkyl-substituted BDT (PBDTI-OD and PBDTI-DT) donor units, while in the third copolymer (PTI-DT), an alkyl-substituted thiophene unit is used. The structures of the BDT-based copolymers were systematically tailored by increasing the side chain length in the isoindigo units from 2-octyldodecyl (OD) in PBDTI-OD to 2-decyltetradecyl (DT) in PBDTI-DT, while keeping the backbone structure the same. The third copolymer, PTI-DT, was synthesized to study the effect of electron-donating groups by changing the donor units from BDT to thiophene. The structural differences in the three copolymers allowed us to investigate the effect of backbone structure on their photo-physics, thermal and structural properties. We found two relaxation channels in the BDT-based copolymers, while a one-channel relaxation was found in PTI-DT. The copolymers showed excellent thermal stability with decomposition temperatures $\left(\mathrm{T}^{D}=5 \%\right.$ mass loss) above $380^{\circ} \mathrm{C}$. The X-ray diffraction patterns of the copolymers suggested that a better structural ordering was found in the thiophene-based copolymer.

\section{Materials and Methods}

\subsection{Materials and Synthesis}

Three copolymers based on an isoindigo acceptor were designed and synthesized using a direct heteroarylation polymerization (DAP) method for the first time. The two copolymers were synthesized by the copolymerization of a 4,8-bis(decyl)benzo[1,2-b;4,5$\mathrm{b}^{\prime}$ ]dithiophene (BDT) (1) donor unit and an isoindigo acceptor unit. N-Alkylation of the isoindigo moiety was achieved with 2-octyldecylbromide and 2-decyltetradecylbromide to obtain compounds $\mathbf{2}$ and $\mathbf{3}$ which were used to synthesize PBDTI-OD and PBDTI-DT, respectively, as shown in Scheme 1. In the synthesis of PBDTI-OD/PBDTI-D, a 1:1 ratio of BDT (1) $(0.5 \mathrm{mmol})$ and isoindigo $(2 / 3)(0.5 \mathrm{mmol})$ was copolymerized using palladium acetate, $(0.01 \mathrm{mmol})$, pivalic acid $(0.15 \mathrm{mmol})$ and potassium carbonate $(1.3 \mathrm{mmol})$ as the catalyst, acidic additive and base, respectively. The polymerization reaction took place 
in an inert atmosphere at a temperature of $100{ }^{\circ} \mathrm{C}$ using anhydrous dimethylacetamide (DMAc) for PBDTI-DT and a mixture of DMAc and toluene as the reaction solvents for PBDTI-OD. The heating was stopped after 7 and $19 \mathrm{~h}$ for PBDTI-OD and PBDTI-DT, respectively, and the solutions were left to cool down to room temperature. The copolymers were precipitated in methanol and filtered. The crude copolymers were dissolved in chloroform (CF) and washed with aqueous solution of ethylene-diamine tatraacetic acid (EDTA) $(\mathrm{pH}=8)$ to remove trace metals, followed by $0.1 \mathrm{~N} \mathrm{HCl}$ and distilled water. PBDTIOD was Soxhlet-extracted with methanol, hexane, diethylether and chloroform while PBDTI-DT was extracted with methanol, acetone, ethylacetate, hexane, diethylether and chloroform. The CF extract of PBDTI-OD was concentrated and precipitated in acetone and filtered. After drying the polymer at $40{ }^{\circ} \mathrm{C}$ in a vacuum oven for $24 \mathrm{~h}$, the solid obtained was further stirred in hexane overnight and filtered to obtain $0.36 \mathrm{~g}$ blue-black copolymer (56\%). Similarly, the CF extract of PBDTI-DT was concentrated precipitated in methanol, filtered, dried in an oven at $40{ }^{\circ} \mathrm{C}$ for $24 \mathrm{~h}$ to afford $0.32 \mathrm{~g}$ dark brown solid $(43 \%)$.

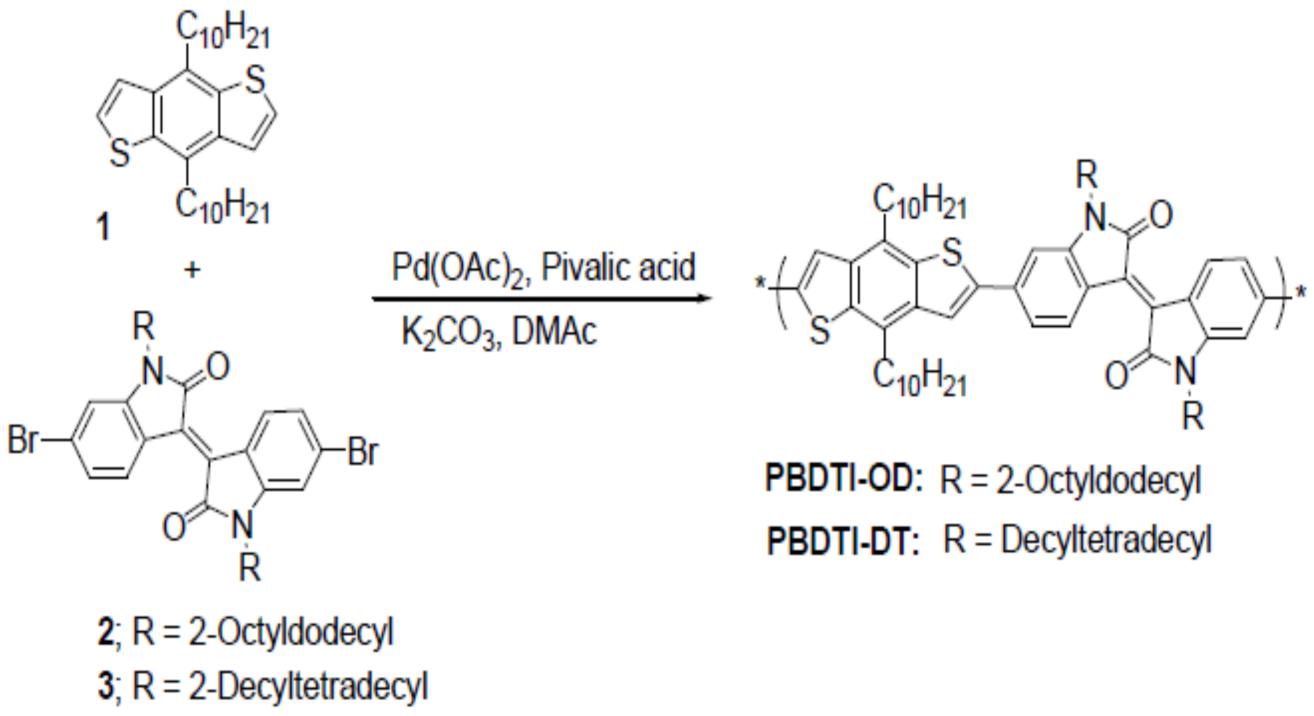

Scheme 1. Syntheses scheme of PBDTI-OD and PBDTI-DT.

PTI-DT was synthesized by copolymerizing 3-octylthiophene (4, $0.5 \mathrm{mmol})$ with isoindigo (3), as shown in Scheme 2. The polymerization was done in anhydrous DMAc for $3.5 \mathrm{~h}$. Following the same procedure, the crude polymer was extracted. PTI-DT was Soxhlet-extracted in methanol, acetone, ethyl acetate and CF, which afforded $0.37 \mathrm{~g}(66 \%)$ dark-brown solid after drying in an oven at $40{ }^{\circ} \mathrm{C}$ overnight. Note here that the long side chains attached to both the donor and acceptor units of the copolymers increased their solubilities in chlorinated solvents like CF and 1,2-dichlorobenzene (oDCB). Comparing the three copolymers, PBDTI-OD was found to be less soluble in these chlorinated solvents like CF and oDCB. On the other hand, introduction of the long side chains might create steric hindrance that might twist the backbones of the copolymers. However, BDT has a planar structure that is expected to make the backbones of PBDTI-OD and PBDTI-DT less twisted and have a longer conjugation length than the thiophene-based copolymer, PTI-DT [12,13]. 


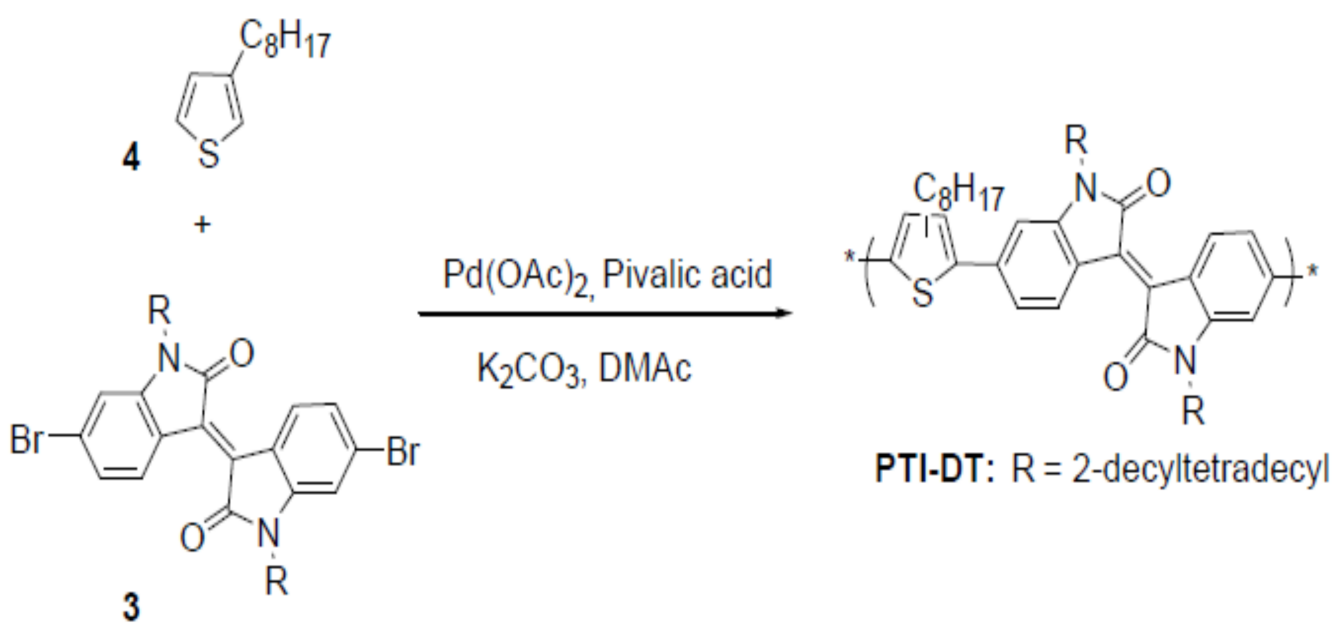

Scheme 2. Synthesis scheme of PTI-DT.

The molecular weights of the copolymers weree determined by size exclusion chromatography was done using a Waters Alliance GPVC2000 instrument with a refractive index detector. The columns used were Waters Stragel ${ }^{\circledR}$ HMW 6EX2 and Waters Stragel ${ }^{\circledR}$ HT 6EX1 and the measurements were taken in 1,2 4-trichlorobenzene at a temperature of $135^{\circ} \mathrm{C}$. The relative molecular mass was measured against polystyrene standards. The average molecular weights $\left(\mathrm{M}_{w}\right)$ of PBDTI-OD, PBDTI-DT and PTI-DT were found to be $53,461,71,234$ and $23,574 \mathrm{~g} / \mathrm{mol}$, respectively. The high $\mathrm{M}_{w} \mathrm{~s}$ of the copolymers are due to the long alkyl side chains appended both in the donor and acceptor units. The calculated poly-dispersity indices (PDI $=\mathrm{M}_{w} / \mathrm{M}_{n}$ ) of the three copolymers show that PTI-DT has a more uniform molecular weight distribution. The molecular weight and yield data are summarized in Table 1.

Table 1. Molecular weight and yield data of PBDTI-OD, PBDTI-DT and PTI-DT.

\begin{tabular}{lcccc}
\hline Copolymer & Yield (\%) & $\mathbf{M}_{n}(\mathbf{g} / \mathbf{m o l})$ & $\mathbf{M}_{w}(\mathbf{g} / \mathbf{m o l})$ & PDI \\
\hline PBDTI-OD & 56.25 & 16,932 & 53,461 & 3.2 \\
PBDTI-DT & 43.71 & 17,974 & 71,234 & 4.0 \\
PTI-DT & 66.07 & 12,537 & 23,574 & 1.9 \\
\hline
\end{tabular}

\subsection{Absorption and Photoluminescence Spectroscopy}

The absorption spectra of PBDTI-OD, PBDTI-DT and PTI-DT in both solutions and spin-coated films were recorded using a Perkin Elmer Lambda 19, UV/Vis/NIR spectrophotometer. The thin films were spin-coated from a CF solution of concentration $10 \mathrm{mg} / \mathrm{mL}$ at a spin speed of $1000 \mathrm{rpm}$ and the films were annealed at $85^{\circ} \mathrm{C}$ for $10 \mathrm{~min}$. Similarity, their photoluminescence (PL) spectra were recorded both in solutions and spin-coated films using JY Horiba, 'Fluoromax'-4 spectrofluorometer. The absorption and PL spectra in solutions were taken in CF, o-DCB, and cyclohexane (Chex) with polarity indices of 4.1, 2.7 and 0.2, respectively. The PL spectra of the copolymers in solutions and films were taken at excitations matching their longer wavelength electronic transition. The solutions for PL measurement absorb only 0.05 at the maxima to avoid self absorption.

\subsection{X-ray Diffraction}

The X-ray diffraction (XRD) patterns of the copolymers were recorded for for $2 \theta$ values from 2 to $40^{\circ}$ in drop-casted films on a glass substrates using Shimadzu Scientific Instruments X-ray diffractometer $7000 \mathrm{~S}$ with a copper target, $\mathrm{Cu}-\mathrm{K} \alpha$-radiation with 
$40 \mathrm{kV} \times 40 \mathrm{~mA}$ power. The interlayer spacing between the planes $(d)$ was calculated using Bragg's Equation (1).

$$
n \lambda=2 d \sin (\theta),
$$

where $n, \lambda$ and $\theta$ are order of diffraction (1), wavelength of the X-ray $(0.154 \mathrm{~nm})$ and angle of diffraction, respectively.

\subsection{Thermogravimetric Analysis}

The thermal properties of the copolymers were investigated using DTG-60H thermogravimetric analyser in platinum crucibles under nitrogen atmosphere flowing at a rate of $50 \mathrm{~mL} / \mathrm{min}$.

\subsection{Electrochemistry}

Square wave voltammetry (SWV) was done to measure the frontier molecular orbitals of the copolyers in a three-electrode setup. Platinium electrodes were used both as working and counter electrodes, while $\mathrm{Ag} / \mathrm{AgCl}$ was used as a reference electrode, which was calibrated against ferocene/ferrocynium ion $\left(\mathrm{FC}_{\mathrm{C}} / \mathrm{FC}^{+}\right)$. The redox potential of $\mathrm{Fc} / \mathrm{Fc}^{+}$was found to be $0.44 \mathrm{eV}$ versus the reference electrode, taking its energy level to be $4.8 \mathrm{eV}$ below the vacumm. The copolymer films were drop-casted on the $\mathrm{Pt}$ disc working electrode, and a $0.1 \mathrm{M}$ solution of tetrabutylammonium hexafluorophosphate $\left(\mathrm{Bu}_{4} \mathrm{NPF}_{6}\right)$ in anyhdrous acetonitrile served as a supporting electrolyte. The higherst occupied molecular orbital (HOMO) and lowest unoccupied molecular orbital (LUMO) energy levels were determined from the onsets of oxidation and reduction potentials, respectively, using Equation (2) [14].

$$
E_{\text {HOMO } / \text { LUMO }}=e\left(E_{\text {ox } / \text { red }}+4.4\right) e V
$$

\section{Results and Discussion}

\subsection{Optical and Electrochemical Properties of PBDTI-OD, PBDTI-DT and PTI-DT}

The absorption spectra of PBDTI-OD, PBDTI-DT and PTI-DT were taken both in CF solutions and as thin films, as shown in Figure 1a,b. The spectra of PBDTI-OD and PBDTI-DT in CF solutions are characterized by one dominant transition peaking at 631 and $628 \mathrm{~nm}$, respectively, and two peaks below $450 \mathrm{~nm}$. On the other hand, the absorption spectrum of PTI-DT is quite different from the BDT-based copolymers where a dominant one transition in solution that peaks at $615 \mathrm{~nm}$ and a modest peak at $450 \mathrm{~nm}$ are observed. The two-energy-band profile exhibited in PBDTI-OD, PBDTI-DT and PTI-DT is common in push-pull copolymers. The low energy band is due to the $S_{0} \rightarrow S_{1}$ transition, usually with intra-molecular charge transfer (ICT) characteristics due to the charge transfer between the donor and acceptor moieties in the copolymers. On the other hand, the high energy band is due to the $\pi-\pi^{*}$ transition, which is common in the $\pi$-conjugated polymers due to the alternating single and double bonds in their backbone [15]. The presumably assigned ICT state transition in PBDTI-OD and PBDTI-DT is red-shifted by more than $11 \mathrm{~nm}$ with respect to PTI-DT, suggesting a better electron-donating property of the BDT moiety [16]. This could also be due to the long alkyl side chains on the thiophene and isoindigo units of PTI-DT that can sterically intract, leading to a twisting in the backbone of the copolymer. Computaion analusis of a similar BDT-based copolymer showed that the copolymers are likely to adopt a planar structure [16] which facilitates ICT in PBDTI-OD and PBDTI-DT chains. 

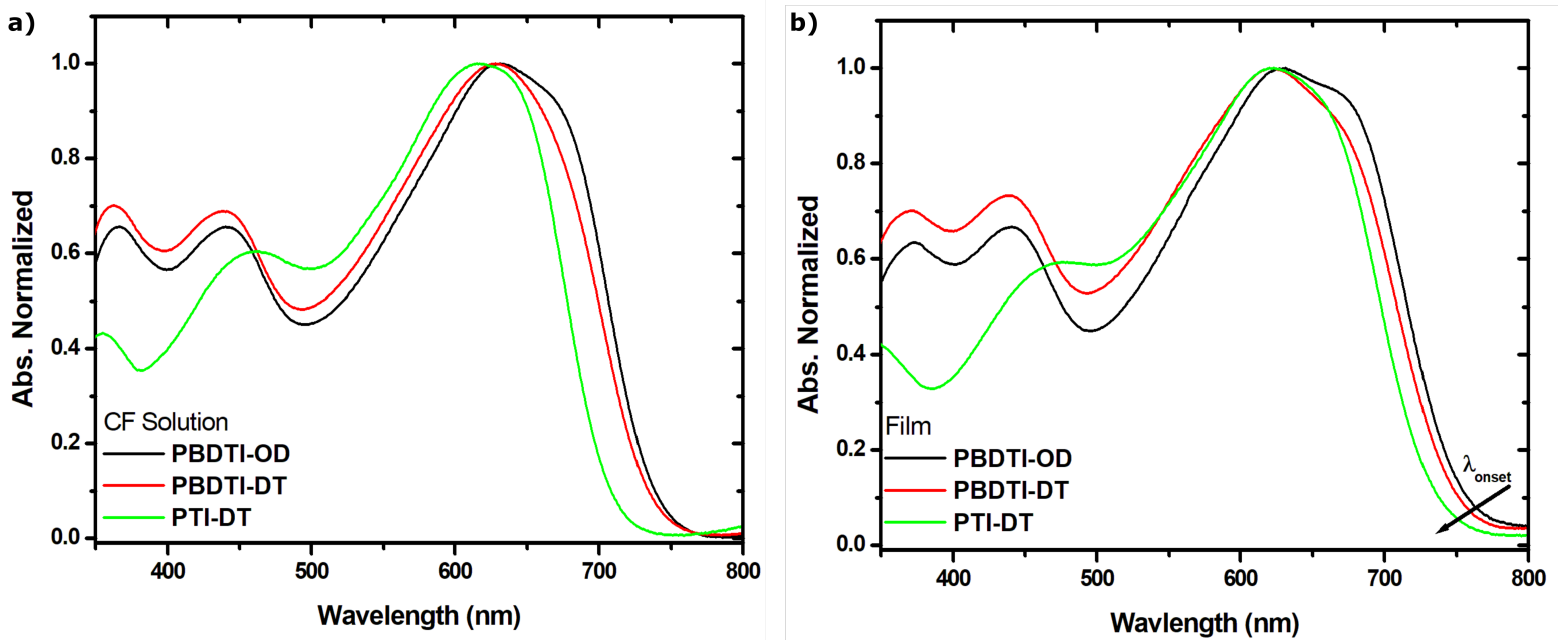

Figure 1. Absorption spectra of PBDTI-OD (black), PBDTI-DT (red) and PTI-DT (green) in (a) CF solutions and (b) thin films.

The absorption spectra of thin films of PBDTI-OD, PBDTI-DT and PTI-DT were found to be red-shifted by 30, 25 and $40 \mathrm{~nm}$ from their solution spectra, respectively. The red-shift in absorbance upon solidification shows a better interchain interaction in the films due to $\pi-\pi$ stacking, which is beneficial for charge transport when the copolymers are used in OSCs. The larger red-shift in absorbance of PTI-DT upon solidification indicates that a different intra- and intermolecular interaction from the DBT-based copolymers exists due to the thiophene donor unit. One of the reasons for this could be that PTI-DT is twisted in the absence of strong intermolecular interaction in dilute solution. However, the stronger inter-molecular force in the solid films pushes the copolymer into a planar conformation, resulting in a siginicant red-shift in absorption onset $[17,18]$. Similarly, the smaller absorption onset shift in the films of PBDTI-DT compared to PBDTI-OD shows the more planar structure of PBDTI-OD due to the shorter side chain on the isoindigo unit [19]. The optical band gaps $\left(\mathrm{E}_{g}\right)$ of the three copolymers were calculated from the onsets of absorption spectra of the films and were found to be 1.53, 1.54 and $1.56 \mathrm{eV}$ for PBDTI-OD, PBDTI-DT, and PTI-DT, respectively. The copolymers have a similar bandgap, that is suitable to harvest substantial solar irradiation in the high solar flux region [20]. The backbone structures of the copolymers have negligible effect on their band gaps. The optical properties of the copolymers are summarized in Table 2.

Table 2. Electrochemical and Optical Properties of PBDTI-OT, PBDTI-DT and PTI-DT.

\begin{tabular}{|c|c|c|c|c|c|c|c|c|c|}
\hline \multirow[t]{2}{*}{ Name } & \multicolumn{2}{|c|}{$\lambda_{\max } \operatorname{Soln}(\mathrm{nm})$} & \multicolumn{2}{|c|}{$\lambda_{\max }$ Film (nm) } & \multicolumn{2}{|c|}{${ }^{1}$ Shift (nm) } & \multirow{2}{*}{$\frac{{ }^{2} E_{g}{ }^{O p t}}{\mathrm{eV}}$} & \multirow{2}{*}{$\frac{E_{\text {НОМо }}}{\mathrm{eV}}$} & \multirow{2}{*}{$\frac{E_{\text {LUMO }}}{\mathrm{eV}}$} \\
\hline & $\lambda_{\max 1}$ & $\lambda_{\max 2}$ & $\lambda_{\max 1}$ & $\lambda_{\max 2}$ & $\Delta \lambda_{\max 1}$ & $\Delta \lambda_{\max 1}$ & & & \\
\hline PBDTI-OD & 366,440 & 631 & 374,440 & 631 & 8,0 & 0 & 1.53 & -5.5 & -3.9 \\
\hline PBDTI-OT & 363,437 & 628 & 370,438 & 623 & 7,4 & -5 & 1.54 & -5.7 & -3.8 \\
\hline PTI-DT &,- 457 & 615 &,- 461 & 623 &,- 4 & 8 & 1.56 & -5.6 & -3.7 \\
\hline
\end{tabular}

The PL spectra of the thin films were recorded by exciting at their absorption maxima in the longer wavelength region and were found to be Stokes-shifted by 179, 189 and $185 \mathrm{~nm}$ in PBDTI-OD, PBDTI-DT and PTI-DT, respectively (see Figure 2). Since an efficeint overalp between the absorption and emission spectra of the copolymers is needed for Förster resonance energy transfer (FRET), the large Stokes shift in the copolymers will inhibit interchain excitation energy transfer [21]. However, the large Stokes shift also shows that multiple processes have taken place to stabilize the first excited state. Since ICT formation in solid films is not efficient, the emission from the first excited state $\left(S_{0} \leftarrow S_{1}\right)$ is ascribed to tightly bound intrachain exciton relaxation. The strong $\pi-\pi$ interaction upon 
solidfication of the copolymers enables an efficient interchain charge transfer interaction that dominates over intrachain charge transfer (i.e., ICT population). Our work on three similar copolymers with two and three thiophene donor units, coupled with an isoindgo acceptor unit, showed similar results $[7,8]$.

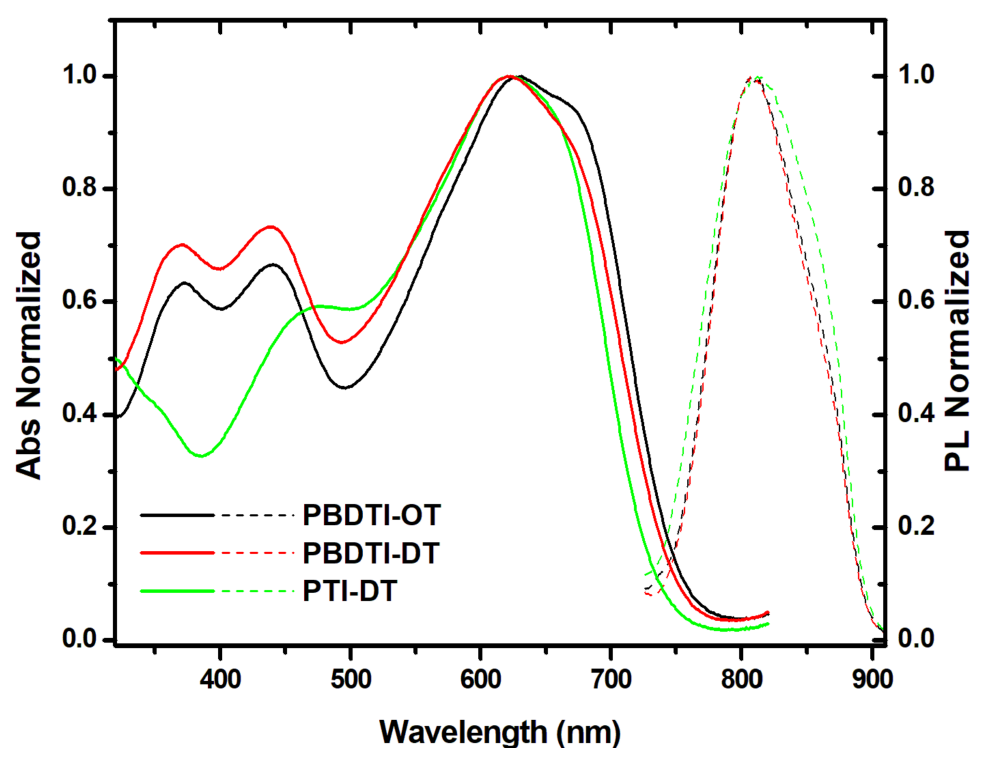

Figure 2. Absorption (solid line) and PL (broken line) spectra of PBDTI-OD (black), PBDTI-DT (red) and PTI-DT (green).

The HOMO and LUMO energy levels of the copolymers were determined using SWV from the onsets of their oxidation and reduction potentials, as shown in Figure 3. The HOMO and LUMO energy levels of the copolymers are summarized in Table 2. The difference in the HOMO levels between the copolymers with the same donor unit (PBDTI-OD and PBDTI-DT) was $0.2 \mathrm{eV}$, whereas the difference is only $0.1 \mathrm{eV}$ between PBDTI-DT and PTI-DT (with the same acceptor unit). The highest LUMO level was found in PBDTI-OD, followed by PBDTI-OD and PTI-DT. The $\pi$-electron density in the copolymers is not expected to be significantly affected by the alkyl side chains [22]. The reason for the difference in HOMO levels between PBDTI-OD and PBDTI-DT should, therefore, be due to the effect of the length of the alkyl side chains on the reorganization of the copolymers in the drop-casted films $[8,19]$. This is expected due to the relatively lower solubility of PBDTI-OD. 


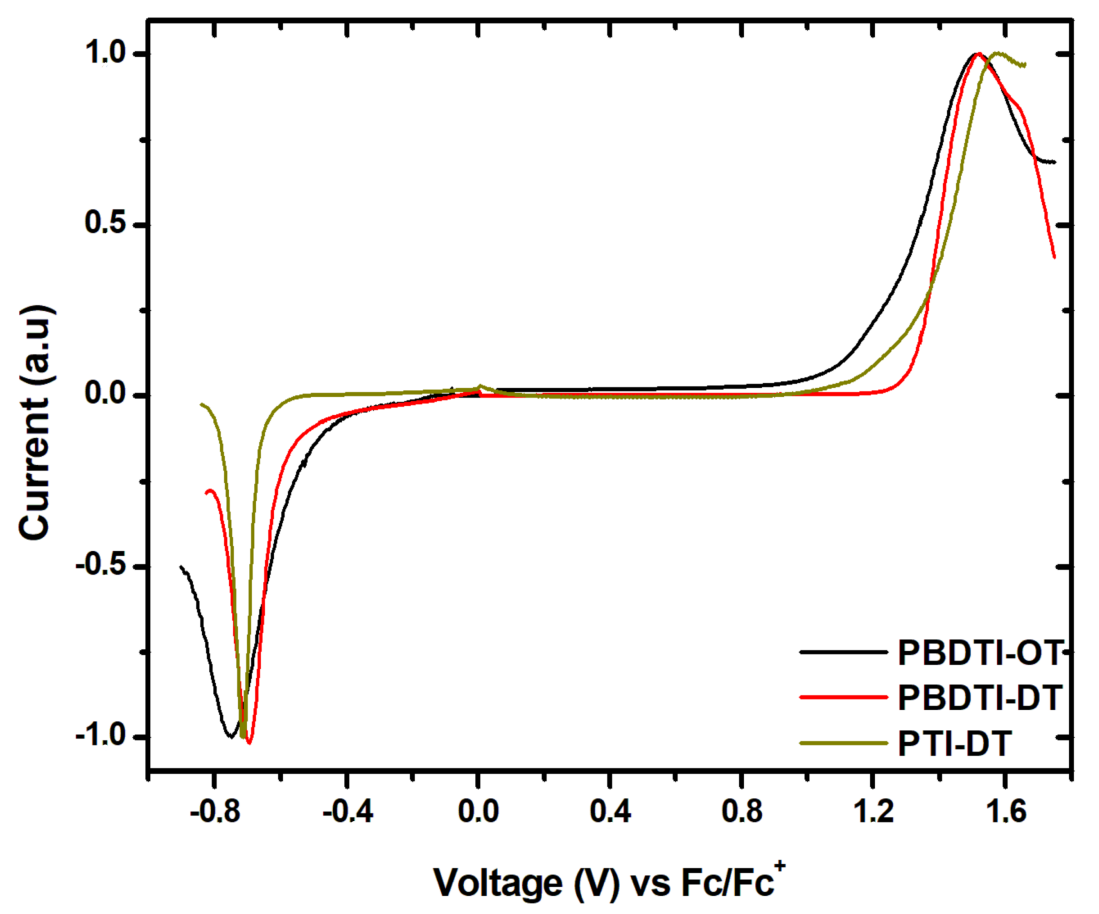

Figure 3. Square wave voltammograms of PBDTI-OD (black), PBDTI-DT (red) and PTI-DT (dark yellow).

The difference in the energetics of PBDTI-DT and PTI-DT is significantly dependent on the electron-donating strength of BDT vesus thiophene units, respectively. The stronger electron-donating property of BDT would enhance the ICT in PBDTI-DT, which will stabilize its LUMO level compared to PTI-DT, in which the donor unit is a thiophene [23]. Note here that the reorganization of the copolymers in the casted films also plays a role. The medium-lying HOMO levels of the copolymers are beneficial for attaining high opencircuit voltage when they are used in OSCs as donor materials, since $V_{O C}=e\left(E_{\text {LUMO }}^{\text {Acceptor }}\right.$ $\left.E_{H O M O}^{\text {Donor }}\right)$, where $e$ is the elementary charge [24]. The LUMO offset of the copolymers with a fullerene acceptor should be around $0.3 \mathrm{eV}$ for efficient exciton disscociation at the donor/acceptor interface [24,25]. PBDTI-OD, PBDTI-DT and PTI-DT have LUMO offsets of $0.1,0.2$ and $0.3 \mathrm{eV}$, respectively with respect to the commonly used fullerene acceptor, $\mathrm{PC}_{71} \mathrm{BM}(\mathrm{LUMO}=4.0 \mathrm{eV})$. The LUMO offsets in the BDT-based copolymers are below the recommended value, which is detrimental to exciton dissociation in fullerene-based OSCs, while efficient exciton dissociation is expected in PTI-DT-based OSCs. Hence, PBDTI-OD and PBDTI-DT might work better when blended with non-fullerene acceptors.

\subsection{Photophysical Properties of the Copolymers}

To further understand the photophysics of the copolymers, their absorption and PL spectra were recorded in $\mathrm{CF}, \mathrm{ODCB}$ and Chex solution in a concentration range of $125-5.7 \mu \mathrm{g} / \mathrm{mL}$. The absorbances of all the copolymers in the three solvents showed negligible shift with concentration gradient, which indicates the absence of aggregation in the solutions. The absorption spectra of the copolymers in the three solvents of different polarity are almost similar, with a slight difference in the apperance of a shoulder when the poor solvent (Chex) was used. However, the low-energy absorption peaks of the copolymers remained the same with increasing solvent polarity, as shown in Figure 4a-c (solid line). This confirms that the ground states $\left(\mathrm{S}_{0}\right)$ of the copolymers are non-polar. The PL spectra of PBDTI-OD showed a red-shift of $18 \mathrm{~nm}$ as the solvent polarity increased from 2.7 (oDCB) to 4.2 (CF). The Stokes shift of PBDTI-OD in Chex is higher than both $\mathrm{oDCB}$ and $\mathrm{CF}$, despite its low polarity index of 0.2. Similarly, a $29 \mathrm{~nm}$ red-shift in PL of PBDTI-DT is found with increasing solvent polairty from 2.7 (oDCB) to 4.2 (CF). An ever 
larger Stokes shift in was observed when Chex was used as a solvent. The PL spectra of PTI-DT showed negligible shifts in the three solvents.
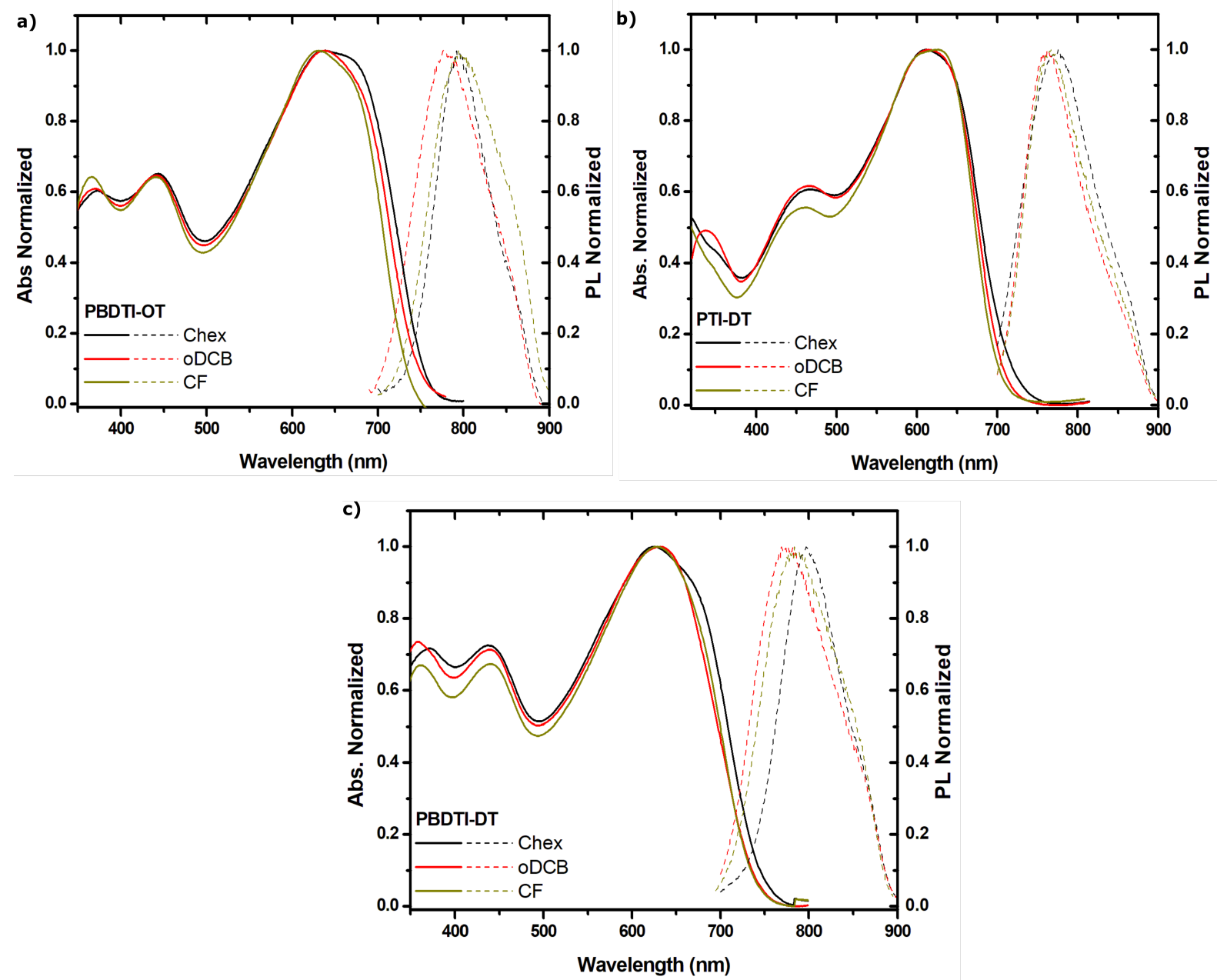

Figure 4. Absorption (solid line) and PL (broken line) of (a) PBDTI-OT, (b) PBDTI-DT and (c) PTI-DT in Chex (black), oDCB (red) and CF (dark yellow) solutions.

The red-shift in PL of PBDTI-OD and PBDTI-DT with increasing solvent polarity while their absorption remained the same confirms the higher dipole moments of their first excited states $\left(\mathrm{S}_{1}\right)$. This is consistent with our assumption that the longer wavelength region transitions have ICT characterers. Hence, their electronic structure changes from the nonpolar $D-A$ to the dipolar $D^{+}=A$ - configuration [15]. The larger Stokes shift observed in Chex solutions of PBDTI-OD and PBDTI-DT shows the population of a different state than ICT. Consequently, the electrons in the first excited state of the copolymers have two channels; one is the ICT state and the other is the tightly bound excitonic state. The latter is populated when there is a significant interchain interaction either in films or solutions. A poor solvent like Chex is expected to form aggregates that induce interchain interaction in the solutions, resulting in red-shifted PL. Thus, the emission in the non-polar solvent could be explained by the tightly bound interchain exciton model instead of the commonly used ICT model [26,27]. However, the Uv-Vis absorbances of PBDTI-OD and PBDTIDT taken in solutions of similar concentration, as the PL measurements confirmed the absence of aggregation. Therefore, the aggregation effect observed in the Chex solutions should be a self-aggregation, causing the copolymer chains to form tight coils. As a result, the excited electrons could be transferred to the other part of the chain acting as interchain excitons $[7,28]$. Consequently, the PL spectra were red-shifted despite the low polarity of the solvent. 
The photophysics of PTI-DT is quite different in that both its ground and exited states are not influenced by the polarities of the solvents. The similar Stokes shift, both in polar (CF and oDCB) and the non-polar (Chex) solvents, shows that self-aggregation of the copolymer chain exists in all solvents. PTI-DT showed a large absorption onset shift as well as a peak shift, while the BDT-based copolymers showed no peak shift upon solidification (see Table 2). This can be attributed to the twisting of the thiophene-based copolymer in dilute solution in the absence of a strong intermolecular interaction. The twisting in the backbone of PTI-DT favours the self-aggregation effect in any solvent. The ICT population from the first excited state of PTI-DT is dominated by the excitonic state, making the emitting state insensitive to polarity of the environment.

In summary, the BDT-based copolymers have a bi-relaxation channel that can be modulated with the solvents, while PTI-DT has a quasi-one-relaxation channel. The population of the ICT and tightly bound excitonic states were found in the BDT-based copolymers, while only the latter was found in PTI-DT. The results confirm the importance of donor units in the synthesis of D-A copolymers for efficient ICT processes.

\subsection{Thermal Properties of PBDTI-OT, PBDTI-DT and PTI-DT}

The thermal properties of PBDTI-OT, PBDTI-DT and PTI-DT were studied using TGA, as shown in Figure 5. The three copolymers have agood thermal stabilities, with decomposition temperatures $\left(\mathrm{T}^{D}=5 \%\right.$ weight loss) above $380{ }^{\circ} \mathrm{C}$. The thermal degradation in these materials follows two steps: one around $380{ }^{\circ} \mathrm{C}$ and the other above $550{ }^{\circ} \mathrm{C}$. The first degradation accounts for the breaking up of the side chains. The shorter side chain in the isoindigo unit of PBDTI-OT slightly increased its thermal stability compared to PBDTI-DT, despite its lower molecular weight. The second thermal degradation is due to the decomposition of the backbone structures of the copolymers. The slight increase in the degradation temperature in PBDTI-OT also shows the strong bonding in its backbone structure compared to PBDTI-DT and PTI-DT. In general, the thermal stabilities of the copolymers make them applicable to flexible OSCs, which are normally processed at temeratures below $150^{\circ} \mathrm{C}$.

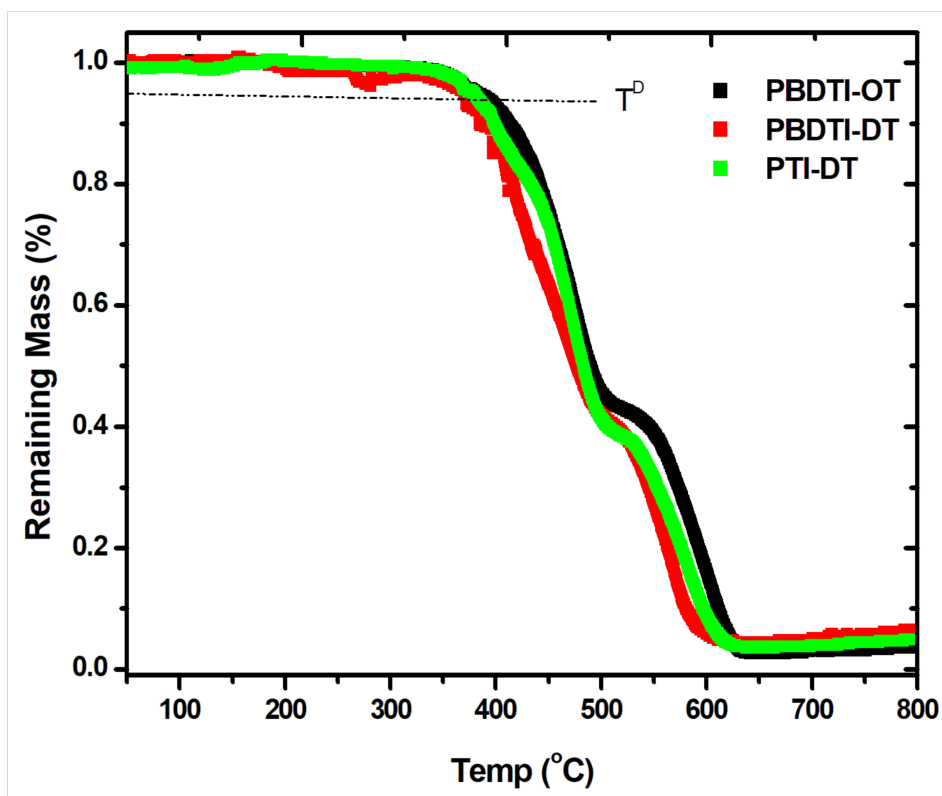

Figure 5. TGA measurement of PBDTI-OT (black), PBDTI-DT (red) and PTI-DT (green). 


\subsection{Structural Properties of PBDTI-OT, PBDTI-DT and PTI-DT Films}

The structures of the copolymers were studied by powder XRD in drop-casted thin films, and the results are shown in Figure 6. The XRD traces of the three copolymers exhibit a broad peak (010) centered around $2 \theta=22^{\circ}$ due to the $\pi-\pi$ stacking.

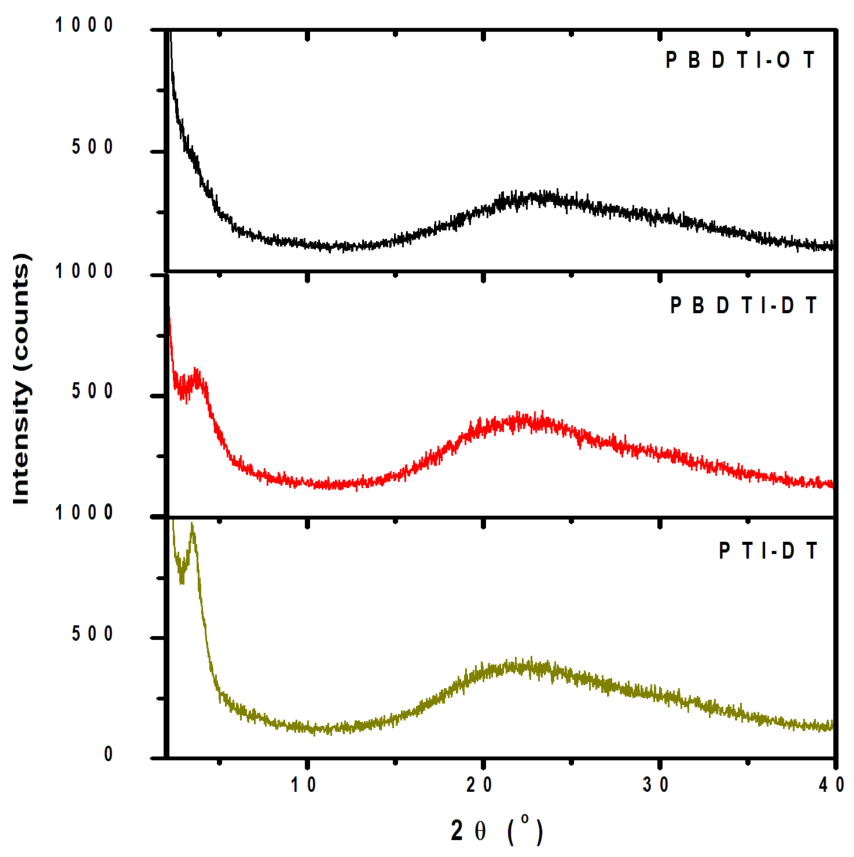

Figure 6. XRD traces PBDTI-OT (black), PBDTI-DT (red) and PTI-DT (dark yellow) in drop-casted films.

The calculated $\pi-\pi$ stacking distances using Bragg's equation Equation (1) are 0.38, 0.41 and $0.41 \mathrm{~nm}$ in PBDTI-OT, PBDTI-DT and PTI-DT films, respectively, confirming the presence of strong intermolecular interaction in the copolymer backbones [29]. PBDTDT and PTI-DT exhibit a (100) diffraction peak at $2 \theta=3.82^{\circ}$ and $3.60^{\circ}$, corresponding to lammelar spacings of 2.3 and $2.5 \mathrm{~nm}$, respectively. The inter-lammelar spacing in PTI-DT $(2.5 \mathrm{~nm})$ is higher than the well-studied thiophene-based homopolymer, poly3-hexylthiophne (P3HT) $(\mathrm{d}=1.63)$ [30]. This is due to the longer side chains both in the thiophene and isoindigo units of PTI-DT compared to P3HT. Note here that the (100) plane diffraction peak is sharper in PTI-DT, indicating its slightly higher crystallinity over the BDT-based copolymer. On the other hand, the BDT-based copolymer with a shorter side chain on the isoindigo unit (PBDT-OT) is fully amorphous. The higher crystallity of thiophene-based copolymer is beneficial for improved charge transport.

\section{Conclusions}

Three copolymers with BDT (PBDTI-OD and PBDTI-DT) and thiophene (PTI-DT) donor units and isoindigo acceptor units were designed and synthesized using the DAP method. The molecular weights of the BDT-based copolymers were more than two-fold higher than that of PTI-DT. The copolymers absorb in a wide range from from 300 to above $760 \mathrm{~nm}$ in thin films, making them low-band-gap polymers with $\mathrm{E}_{g} \approx 1.5 \mathrm{eV}$. The photophysical properties of the copolymers were studied in both films and solutions. Solvent polarity-dependent spectroscopic studies on PBDTI-OD and PBDTI-DT showed that the ground state is not sensitive to polarity of the environment, while the excited state is. This led us to conclude that the ground states of the BDT-based copolymers are non-polar. The PL spectra of the BDT-based copolymers were red-shifted with increasing solvent polarity of chlorinated solvents, confirming the dipolar characteristics of their first excited states. Therefore, the first excited state has ICT characteristics. However, the Stokes shift in PBDTI-OD and PBDTI-DT in the non-polar solvent, Chex, was not blue-shifted, as 
expected from its low polarity index. Self-aggregation of the copolymers in the poor solvent was found to favour the population of a tightly bound excitonic state, thereby inhibiting the generation of ICT state. The absorption and PL spectra of the thiophene-based copolymer were not influenced by solvent polarity. This led us to conclude that self-aggregation in the thiophene-based copolymer is induced despite the solvent polarity. Therefore, ICT state population in PTI-DT is not efficient. The copolymers showed excellent thermal stability, with a decomposition temperature above $380^{\circ} \mathrm{C}$. The XRD patterns of the three copolymers showed a halo from (010) plane due to $\pi-\pi$ stacking. A second diffraction peak was observed in PBDTI-DT and PTI-DT, confirming a better crystallinity in these copolymers.

Author Contributions: Z.A. and W.M. synthesized the copolymer and wrote the synthesis part of the manuscript. N.A.T. characterized the polymers and part of wrote the manuscript. All authors have read and agreed to the published version of the manuscript.

Funding: This research was funded by UNESCO and the international Development research Center, Ottawa, Canada, grant number 4500406703.

Institutional Review Board Statement: Not applicable.

Informed Consent Statement: Not applicable.

Data Availability Statement: Data will be available based on request.

Acknowledgments: This work was carried out with the aid of a grant from UNESCO and the international Development research Center, Ottawa, Canada. The views expressed herein do not necessarily represent those of UNESCO, IDRC or its Board of Governors. Z.A. and W.M. would like to acknowledge the financial support from the international Science Program (ISP), Uppsala, Sweden.

Conflicts of Interest: The authors declare no conflict of interest.

\section{References}

1. Liu, Q.; Jiang, Y.; Jin, K.; Qin, J.; Xu, J.; Li, W.; Xiong, J.; Liu, J.; Xiao, Z.; Sun, K.; et al. 18\% Efficiency organic solar cells. Sci. Bull. 2020, 65, 272-275. [CrossRef]

2. Meng, L.; Zhang, Y.; Wan, X.; Li, C.; Zhang, X.; Wang, Y.; Ke, X.; Xiao, Z.; Ding, L.; Xia, R.; et al. Organic and solution-processed tandem solar cells with 17.3\% efficiency. Science 2018, 361, 1094-1098. [CrossRef] [PubMed]

3. Mercier, L.G.; Leclerc, M. Direct (hetero) arylation: A new tool for polymer chemists. Acc. Chem. Res. 2013, 46, 1597-1605. [CrossRef]

4. Saito, H.; Chen, J.; Kuwabara, J.; Yasuda, T.; Kanbara, T. Facile one-pot access to $\pi$-conjugated polymers via sequential bromination/direct arylation polycondensation. Polym. Chem. 2017, 8, 3006-3012. [CrossRef]

5. Rudenko, A.E.; Thompson, B.C. Influence of the carboxylic acid additive structure on the properties of poly (3-hexylthiophene) prepared via direct arylation polymerization (DArP). Macromolecules 2015, 48, 569-575. [CrossRef]

6. Cicha, C.L.; Gockel, S.N.; Helmin, A.J.; Wilcox, W.D.; Janzen, D.E.; Pappenfus, T.M. Benzodithiophene homopolymers via direct (hetero) arylation polymerization. Polym. Bull. 2018, 75, 5667-5675. [CrossRef]

7. Tegegne, N.A.; Abdissa, Z.; Mammo, W.; Andersson, M.R.; Schlettwein, D.; Schwoerer, H. Ultrafast excited state dynamics of a bithiophene-isoindigo copolymer obtained by direct arylation polycondensation and its application in indium tin oxide-free solar cells. J. Polym. Sci. Part B Polym. Phys. 2018, 56, 1475-1483. [CrossRef]

8. Tegegne, N.A.; Abdissa, Z.; Mammo, W.; Uchiyama, T.; Okada-Shudo, Y.; Galeotti, F.; Porzio, W.; Andersson, M.R.; Schlettwein, D.; Vohra, V.; et al. Effect of Alkyl Side Chain Length on Intra-and Intermolecular Interactions of Terthiophene-Isoindigo Copolymers. J. Phys. Chem. C 2020, 124, 9644-9655. [CrossRef]

9. Tegegne, N.A.; Wendimu, H.; Abdissa, Z.; Mammo, W.; Andersson, M.R.; Hone, F.G.; Andoshee, D.M.; Olaoye, O.; Bosman, G. Light-induced degradation of a push-pull copolymer for ITO-free organic solar cell application. J. Mater. Sci. Mater. Electron. 2020, 31, 21303-21315. [CrossRef]

10. Zimmermann, D.; Sprau, C.; Schröder, J.; Gregoriou, V.G.; Avgeropoulos, A.; Chochos, C.L.; Colsmann, A.; Janietz, S.; Krüger, H. Synthesis of D- $\pi$-A- $\pi$ type benzodithiophene-quinoxaline copolymers by direct arylation and their application in organic solar cells. J. Polym. Sci. Part A Polym. Chem. 2018, 56, 1457-1467. [CrossRef]

11. Ye, L.; Zhang, S.; Zhao, W.; Yao, H.; Hou, J. Highly efficient 2D-conjugated benzodithiophene-based photovoltaic polymer with linear alkylthio side chain. Chem. Mater. 2014, 26, 3603-3605. [CrossRef]

12. Liu, Y.; Wan, X.; Wang, F.; Zhou, J.; Long, G.; Tian, J.; Chen, Y. High-performance solar cells using a solution-processed small molecule containing benzodithiophene unit. Adv. Mater. 2011, 23, 5387-5391. [CrossRef]

13. Li, M.; Ni, W.; Wan, X.; Zhang, Q.; Kan, B.; Chen, Y. Benzo [1, 2-b: 4, 5-b'] dithiophene (BDT)-based small molecules for solution processed organic solar cells. J. Mater. Chem. A 2015, 3, 4765-4776. [CrossRef] 
14. Eckhardt, H.; Shacklette, L.; Jen, K.; Elsenbaumer, R. The electronic and electrochemical properties of poly (phenylene vinylenes) and poly (thienylene vinylenes): An experimental and theoretical study. J. Chem. Phys. 1989, 91, 1303-1315. [CrossRef]

15. Jespersen, K.G.; Beenken, W.J.; Zaushitsyn, Y.; Yartsev, A.; Andersson, M.; Pullerits, T.; Sundström, V. The electronic states of polyfluorene copolymers with alternating donor-acceptor units. J. Chem. Phys. 2004, 121, 12613-12617. [CrossRef] [PubMed]

16. Ma, Z.; Wang, E.; Jarvid, M.E.; Henriksson, P.; Inganäs, O.; Zhang, F.; Andersson, M.R. Synthesis and characterization of benzodithiophene-isoindigo polymers for solar cells. J. Mater. Chem. 2012, 22, 2306-2314. [CrossRef]

17. Wang, B.; Zhang, J.; Tam, H.L.; Wu, B.; Zhang, W.; Chan, M.S.; Pan, F.; Yu, G.; Zhu, F.; Wong, M.S. Impact of alkyl side chains on the photovoltaic and charge mobility properties of naphthodithiophene-benzothiadiazole copolymers. Polym. Chem. 2014, 5, 836-843. [CrossRef]

18. Osaka, I.; Saito, M.; Koganezawa, T.; Takimiya, K. Thiophene-thiazolothiazole copolymers: Significant impact of side chain composition on backbone orientation and solar cell performances. Adv. Mater. 2014, 26, 331-338. [CrossRef]

19. Zhang, S.; Ye, L.; Zhao, W.; Liu, D.; Yao, H.; Hou, J. Side chain selection for designing highly efficient photovoltaic polymers with 2D-conjugated structure. Macromolecules 2014, 47, 4653-4659. [CrossRef]

20. Scharber, M.C.; Mühlbacher, D.; Koppe, M.; Denk, P.; Waldauf, C.; Heeger, A.J.; Brabec, C.J. Design rules for donors in bulk-heterojunction solar cells-Towards 10\% energy-conversion efficiency. Adv. Mater. 2006, 18, 789-794. [CrossRef]

21. Yu, Y.; Yang, B.; Yuan, Y.; Zhang, H. Novel side-chain alternative copolymer combined FRET and DRET with large pseudo-Stokes shift and polarity-sensitive fluorescence behavior. J. Mater. Chem. C 2019, 36, 11285-11292. [CrossRef]

22. Tessarolo, M.; Gedefaw, D.; Bolognesi, M.; Liscio, F.; Henriksson, P.; Zhuang, W.; Milita, S.; Muccini, M.; Wang, E.; Seri, M.; et al. Structural tuning of quinoxaline-benzodithiophene copolymers via alkyl side chain manipulation: Synthesis, characterization and photovoltaic properties. J. Mater. Chem. A 2014, 2, 11162-11170. [CrossRef]

23. Zhang, A.; Xiao, C.; Meng, D.; Wang, Q.; Zhang, X.; Hu, W.; Zhan, X.; Wang, Z.; Janssen, R.A.; Li, W. Conjugated polymers with deep LUMO levels for field-effect transistors and polymer-polymer solar cells. J. Mater. Chem. C 2015, 3, 8255-8261. [CrossRef]

24. Brabec, C.J.; Cravino, A.; Meissner, D.; Sariciftci, N.S.; Fromherz, T.; Rispens, M.T.; Sanchez, L.; Hummelen, J.C. Origin of the open circuit voltage of plastic solar cells. Adv. Funct. Mater. 2001, 11, 374-380. [CrossRef]

25. Mola, G.T.; Abera, N. Correlation between LUMO offset of donor/acceptor molecules to an open circuit voltage in bulk heterojunction solar cell. Phys. B Condens. Matter 2014, 445, 56-59. [CrossRef]

26. Sowmiya, M.; Tiwari, A.K.; Saha, S.K. Study on intramolecular charge transfer fluorescence properties of trans-4-[4'-(N, $\mathrm{N}^{\prime}$-dimethylamino) styryl] pyridine: Effect of solvent and pH. J. Photochem. Photobiol. A Chem. 2011, 218, 76-86. [CrossRef]

27. Belletête, M.; Bouchard, J.; Leclerc, M.; Durocher, G. Photophysics and solvent-induced aggregation of 2, 7-carbazole-based conjugated polymers. Macromolecules 2005, 38, 880-887. [CrossRef]

28. Rolczynski, B.S.; Szarko, J.M.; Son, H.J.; Liang, Y.; Yu, L.; Chen, L.X. Ultrafast intramolecular exciton splitting dynamics in isolated low-band-gap polymers and their implications in photovoltaic materials design. J. Am. Chem. Soc. 2012, 134, $4142-4152$. [CrossRef]

29. Choi, M.H.; Song, K.W.; Moon, D.K. Alkylidenefluorene-isoindigo copolymers with an optimized molecular conformation for spacer manipulation, $\pi-\pi$ stacking and their application in efficient photovoltaic devices. Polym. Chem. 2015, 6, 2636-2646. [CrossRef]

30. Vanlaeke, P.; Swinnen, A.; Haeldermans, I.; Vanhoyland, G.; Aernouts, T.; Cheyns, D.; Deibel, C.; D’Haen, J.; Heremans, P.; Poortmans, J.; et al. P3HT/PCBM bulk heterojunction solar cells: Relation between morphology and electro-optical characteristics. Sol. Energy Mater. Sol. Cells 2006, 90, 2150-2158. [CrossRef] 